\title{
Grey Wolf Optimization on Modeling Saliency Detection in Stereoscopic 3D Images
}

\author{
Y Rakesh, K Sri Rama Krishna
}

\begin{abstract}
Till now, several saliency detection models have been introduced for numerous applications in multimedia developments. Anyhow, specific appliances of stereoscopic imaging require improvements in saliency detection schemes for extracting the salient regions in a precise manner. The saliency detection (SD) model faces numerous shortcomings like intricacy in natural images and minor-scale patterns on salient objects. Hence, this paper endeavors to attain the SD model in two levels; Feature extraction (FE), for which Gaussian kernel model is utilized to extort the features and depth SD, for which Gabor Filter (GF) is exploited to attain the depth of saliency map. Accordingly, the adopted scheme optimizes ' 2 ' coefficients such as, feature difference betwixt image patches $H$ in feature evaluation and also fine scale $c$ from which the accurate detection is attained. For optimization purpose, a well-known optimization termed Grey Wolf Optimization (GWO) is exploited and evaluated for varying values of $a$, and the results are attained with respect to ROC (i.e. Receiver Operating Characteristic), and statistical analysis.
\end{abstract}

Keywords - Saliency Detection; Feature extraction; Gaussian Kernel Model; Gabor Filter

\subsection{INTRODUCTION}

According to the human visual observation principle, individuals would like to consign more concentration in the area that stands out from the image backdrop. SD [1] [2] [3] is turning out to be a more significant part in computer visualization owing to its diverse appliance in recognition image retrieval, retargeting, object detection, along with image compression. Normally, SD [4] [5] [6] approaches can well be classified to task-driven top-down model and also a data-driven bottom-up model. SD [7] [8] intends to emphasize salient portions effectively and restrain backdrop areas. Definitely, numerous SD techniques for RGB image

Revised Manuscript Received on July 05, 2019.

Y Rakesh Dept. of ECE,Usha Rama College of Engineering and Technology, Vijayawada, India,

Email: yrk.ur.ece@gmail.com,

K Sri Rama Krishna Dept. of ECE., Velagapudi Ramakrishna Siddhartha Engineering College, Vijayawada, India

Email: kalvasrk@gmail.com.
Definitely, numerous SD techniques for RGB image were presented that combines various visual indications to evaluate the saliency map.

A significant condition that saliency object detection [9] [10] schemes have to gratify is a quicker function, as they operate as a pre-processing phase for ocular data examination intending to lessen the overall load of computation. To sustain this practice as common as feasible, one may desire using unsupervised schemes that do not include the requirement of pre-training in ocular data of the entire probable groupings [11] [12].

Several SD [13][14] techniques were implemented in the previous years that accomplish better performance on uncomplicated datasets, and the development in salient detection remains a rising procedure [15]. Although much development has been accomplished in predicting salient substances [16] [17], most techniques are restricted to the case of detecting uncomplicated scenes that do not entail multifaceted backdrop. However, when a scene comprises of numerous objects with the crowded background, salient approaches do not succeed to obtain equivalent saliency map that is observed by the slower growth on composite datasets [18] [19] [20].

This paper contributes the analysis on SD model in two levels; Feature extraction, for which Gaussian kernel model is deployed to extort the features along with depth SD, for which GF is deployed to attain the depth of saliency map. Consequently, the presented model optimizes ' 2 ' coefficients like, feature difference between image patches, as of feature evaluation together with fine scale, from which the precise detection can well be obtained. For optimization, a renowned optimization model known as GWO is exploited and evaluated for varying values of $a$, and also the outcomes are attained with regard to ROC, and statistical analysis. Xzzx

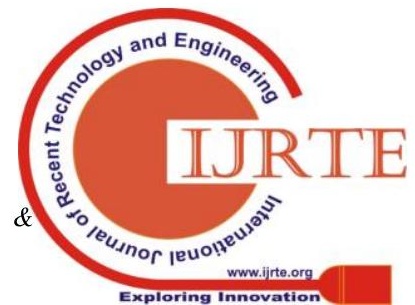




\subsection{LITERATURE REVIEW}

\section{A. Related works}

In 2016, Cong et al. [1] have initially propounded a metric to devise the depth map consistency, and have exploited it on reducing the manipulation on SD. Consequently, the image at input was indicated as a graph, and accordingly, the depth data was established for the construction of the graph. Further, a new description under compression by depth and color signals was forwarded to evaluate the solidity saliency map. At last, the corresponding two saliency maps were incorporated to the last saliency map through the weightedsum approach, which was based upon their importance. The experimentation outcomes have confirmed the superior performance of the suggested work.

In 2017, $\mathrm{Li}$ [2] presented an SD approach relying on the multilayer graph to efficiently identify salient objects in multifaceted prospects. Initially, the targeted position was obtained by exploiting certain prediction algorithms, which was dependent on an inspiration that awareness system could rapidly concern on salient portions prior to processing. Further, by simulating the introduced model, the recognition outcome has offered better results, which most probably includes salient objects that have to be detected.

In 2015, Qing et al [3] recommended a retrieve-based learning algorithm aimed at SD. Here, the adopted scheme was based on consequent observations: diverse models frequently perform inversely, and the performance of the model often differs based on various instances. Therefore, the model of choosing manner was planned to choose the efficient model to a particular instance adaptively. The approach choosing issue has been malformed into an annotation issue that formulates it common for various applications and reliable to work metric learning model.

In 2016, Zhang et al. [4] have developed an algorithm for recognizing the salient regions. When distinguished to other models related to identification in single color space, the developed model pre-segments the particular image that was given as input into superpixels. Next to this, for the formulation of color contrast, the local feature was concerned and also the difference among pixel and the complete image was assessed. Concurrently, centered on center-surrounding approach, a computational technique was developed for detecting the salient regions. At last, 2D entropy was employed as the major evaluation norm for the selection and integration of suitable color features.
In 2016, Diao et al. [5] have projected an effective object detection model, which has combined the potency of the Deep Belief Networks (DBNs). Accordingly, the trained DBN was utilized for FE along with classification on subimages. Moreover, DBN's feature learning was operated via each layer of Restricted Boltzmann Machines (RBMs) by the layer-wise training algorithm. Moreover, an unsupervised approach was introduced for training the $1^{\text {st }}$ layer of RBMs that has merged the raw pixels as inputs. The experimentation was done, and the results demonstrated the accuracy and superiority of the recommended work.

\subsection{FRAMEWORK OF PROPOSED SALIENCY DETECTION}

\subsection{Proposed Archetype}

The diagrammatical demonstration of the implemented SD model is specified in Figure 1. This work aims to implement an SD approach to attain the informative portions obviously as the detection beneath the computer vision is much complex. Here, the input is regarded as the right image $I^{\text {right }}$ as well as a left image $I^{\text {left }}$ as of which the depth features $F^{\text {depth }}$ is extracted by exploiting Gaussian kernel approach. The $F^{\text {depth }}$ features are further processed under GF for improving them to attain the depth saliency map $D^{\text {map }}$, which indicates the 'saliency' at each location on the visual region. Here, two coefficients: feature variation between image patches $U$, from feature assessment, and fine scale $c$, the coefficient of GF is optimized to ensure about the precise SD. For attaining the optimal coefficients, a well-known optimization model known as GWO is deployed. additionally, this work presents meticulous analysis on GWO algorithm, by varying the variable $a$ from 0.5 to 2.5 to check the effectiveness of the respective algorithm on the proposed SD model.

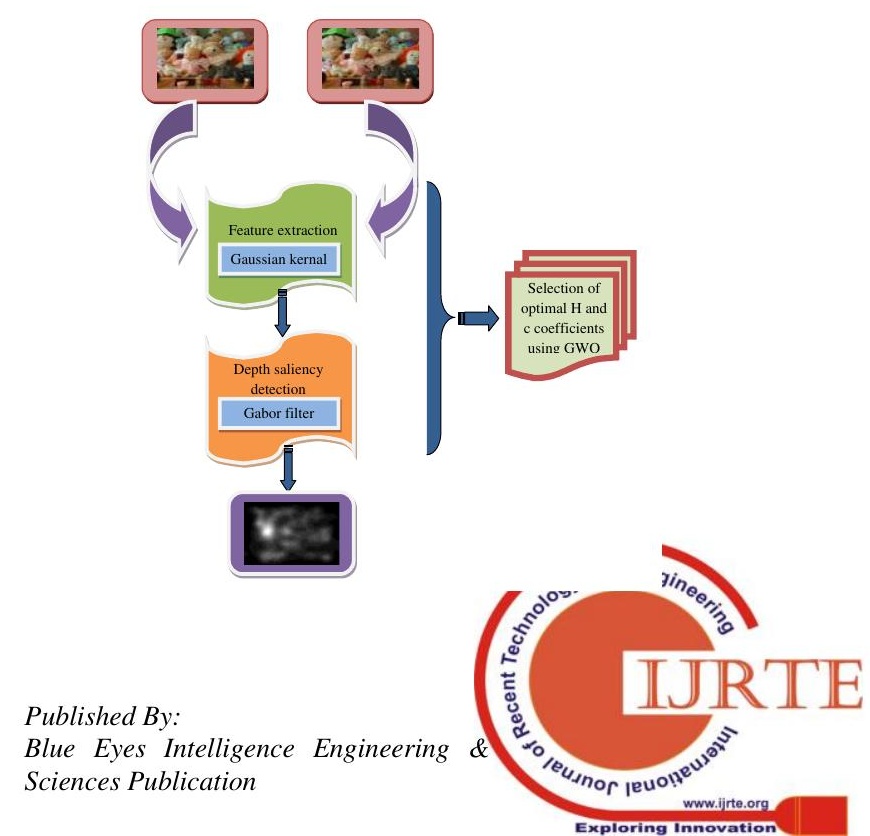


Figure 1: Architecture of Proposed Architecture

\subsection{Gaussian Model}

The process of depth FE is performed using Gaussian model [26] of spatial distance betwixt patches for weighting the contrast of features for assessing the saliency. The saliency value $S_{i}^{f}$ of $i$ image patch from $f$ features is devised as per Eq. (1), in which $f \in\left\{L U, C O_{1}, C O_{2}, T E, F^{\text {depth }}\right\} ; \quad L U$ indicates the Luminance feature, $\mathrm{CO}_{1}$ and $\mathrm{CO}_{2}$ denotes color features, $T E$ specifies the texture feature, $F^{\text {depth }}$ signifies the depth feature, $k_{i j}$ denotes the distance betwixt image patches $i$ and $j ; U_{i j}^{f}$ alludes to the feature variation between $i$ and $j$ from $f, \sigma$ indicates the variable of Gaussian approach that portrays the degree of local and also global contrast.

$$
S_{i}^{f}=\sum_{j \neq i} \frac{1}{\sigma \sqrt{2 \pi}} e^{k_{i j}^{2} /\left(2 \sigma^{2}\right)} H_{i j}^{f}
$$

The evaluation of $H_{i j}^{f}$ is much varied from $f$ as both of them alters in their feature demonstration. Since a single DC coefficient discovers the entire features, the contrast of feature from every features between $i$ and $j$ is assessed as the differentiation between two DC coefficients as given by Eq. (2), in which $D^{n}$ alludes to the feature and also $D^{n} \in\left\{L U, C O_{1}, C O_{2}, F^{\text {depth }}\right\}$; the denominator element is for the normalization of feature contrast.

Accordingly, the feature contrast $\hat{H}_{i j}$ from texture features between $i$ and $j$ is determined as specified in Eq. (3), in which $l$ indicates the AC coefficients and $l \in\{1,2, \ldots .9\}$ refers to the texture feature and the denominator portion is taken as the normalization of feature contrast.

$$
\begin{aligned}
& H_{i j}^{n}=\frac{\left|D_{i}^{n}-D_{j}^{n}\right|}{D_{i}^{n}+D_{j}^{n}} \\
& \hat{H}_{i j}=\frac{\sqrt{\sum_{l}\left(D_{i}^{\prime l}-D_{j}^{\prime \prime}\right)^{2}}}{\sum_{l}\left(D_{i}^{\prime l}+D_{j}^{\prime l}\right)}
\end{aligned}
$$

Thus, the $F^{\text {depth }}$ feature is the attained feature from the FE procedure.

\subsection{Gabor Filter}

The $F^{\text {depth }}$ features attained as of FE are processed under GF to attain the depth saliency map, where the useful information of the input images is available [27]. Here, an intensity image $I N ; I N=(r e+g r+b l)$ is get from green $g r$, red $r e$, and also blue $b l$ channels of the $F^{\text {depth }}$ feature. In addition, Gaussian pyramid of $I N(\sigma)$ is formed by $I N$, in which $\sigma \in[0.8]$ denotes the scale. The indicated channels are generally normalized by $I N$ for the intention of decoupling hue from intensity. Consequently, the formation of generally tuned $R E, \quad B L$ and $G R$ channels is performed; $R E=r e-(g r+b l) / 2$ for red channel, $B L=b l-(r e+g r) / 2$ for blue channel $G R=g r-(r e+b l) / 2$ for green channel and $Y E=(r e+g r) / 2-b l$ for yellow channel. Among those color channels, '4' Gaussian pyramids namely, $R E(\sigma), G R(\sigma), B L(\sigma)$ and $Y E(\sigma)$ are formed.

The depicted center surrounded differences $(-)$ betwixt $c$ 'center' fine scale and $s$ 'surround' coarser scale proffers the feature map as evinced in Eq. (4). The '2' feature sets are formulated: the initial feature map set is established with intensity contrast, where both sensitive kinds are assessed in a six-map set $I N(c, s) ; c \in(2,3,4)$ and $s=c+\delta, \delta \in(3,4)$.

$$
I N(c, s)=|\operatorname{IN}(c)(-) \operatorname{IN}(s)|
$$

Likewise, the formulation for a second map set is executed for color channels. Grounded on this, $\operatorname{REGR}(c, s)$ which stands for green or red and red or green double opponency and $B L Y E(c, s)$ for yellow or blue and blue or yellow double opponency are produced and they are specified by Eq. (5) and Eq. (6).

Here, the local orientation data is attained from $I N$ with the assistance of oriented Gabor pyramids $\operatorname{OR}(\sigma, \theta)$, in which $\theta \in\left\{0^{\circ}, 45^{\circ}, 90^{\circ}, 135^{\circ}\right\}$ indicates the desired orientation and $\sigma \in[0 . .8]$ indicates the scale.

$$
\begin{aligned}
& R E G R(c, s)=\mid(R E(c)-G R(c))(-)(G R(s)-R E(s)) \\
& B L Y E(c, s)=|(B L(c)-Y E(c))(-)(Y E(s)-B L(s))|
\end{aligned}
$$

The depth feature maps, $D^{\text {map }}(c, s, \theta)$ is portrayed as in Eq. (7) that signifies the 
orientation $O R$ contrast between surround scale $S$ and center $c$.

$$
D^{m a p}(c, s, \theta)=|O R(c, \theta)(-) O(s, \theta)|
$$

Consequently, $D^{\text {map }}$ specifies the computed saliency map.

\subsection{SOLUTION ENCODING AND OPTIMAL PARAMETER SELECTION: PROPOSED OBJECTIVE MODEL}

\subsection{Objective Function}

The objective function is portrayed in Eq. (8), in which $O B$ indicates the mean of Pearson Correlation Coefficient (PCC).

$$
O=\max (O B)
$$

\subsection{Solution Encoding}

The optimal assortment of constraints $H$ and $c$ is the utmost significant criteria for the most improved outcomes in the SD of images. This work considers GWO for the attaining the optimal constraints. The solution for the model is demonstrated in Figure. 2.

Accordingly, the solution length $S_{L}$ is specified in Eq. (9), in which length $(H)$ indicates the Gaussian kernel constraint length and length $(c)$ denotes the of GF constraint length.

$$
S_{L}=\text { length }(H)+\operatorname{leng} \operatorname{th}(c)
$$

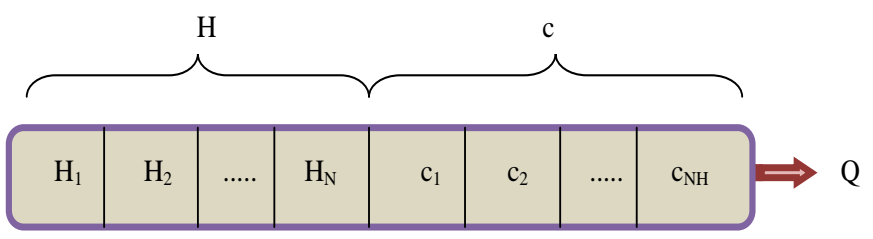

Figure 2: Solution encoding

\subsection{Grey Wolf Optimization}

The optimal assortment of $H$ and $c$ is obtained by means of the GWO algorithm. In general, GWO [31] is a metaheuristic approach, which is dependent on population and it is characterized by the hunting and leadership characteristics of grey wolves. The model includes four varied stages: the initial level is alpha $\alpha$, which acts as the leaders of the troop. They implement decisions concerning walking time, hunting, sleeping place, etc. The subsequent stage known as beta, $\beta$ that assists $\alpha$ in the decision-making process. The third stage is delta $\delta$ that is termed as subordinates. Then the last stage is omega $\omega$ that is regarded as the scapegoat. In this approach $\alpha, \beta$ and $\delta$ assists in the process of hunting.

The encircling behaviour is numerically designed in Eq. (10), in which it stipulates the current iteration, $\vec{B}$ and $\vec{F}$ signifies coefficient vectors, $\vec{Q}_{\text {po }}$ depicts the prey's position vector and $\vec{Q}$ symbolizes the position vector of grey wolf.

$$
\begin{aligned}
& \vec{E}=\mid \vec{F} \cdot \vec{Q}_{p o}(i t)-\vec{Q}(i t) \\
& \vec{Q}(i t+1)=\vec{Q}_{p o}(i t)-\vec{B} \cdot \vec{E}
\end{aligned}
$$

The evaluations of $\vec{B}$ and $\vec{F}$ is portrayed in Eq.(12) and Eq. (13), in which $a$ is minimized linearly from two to zero, $\vec{k}_{1}$ and $\vec{k}_{2}$ signifies the random vectors in the range $[0,1]$.

$$
\begin{aligned}
\vec{B} & =2 a \cdot \vec{k}_{1}-a \\
\vec{F} & =2 \cdot \vec{k}_{2}
\end{aligned}
$$

In general, the hunting procedure is assisted by $\alpha$. The initial ' 3 ' best solutions are attained from the search space, and the update policy is assessed as in Eq. (14), (15) and (16).

$$
\begin{aligned}
& \vec{E}_{\alpha}=\left|\vec{F}_{1} \cdot \vec{Q}_{\alpha}-\vec{Q}\right|, \vec{E}_{\beta}=\left|\vec{F}_{2} \cdot \vec{Q}_{\beta}-\vec{Q}\right|, \vec{E}_{\delta}=\left|\vec{F}_{3} \cdot \vec{Q}_{\delta}-\vec{Q}\right| \\
& \vec{Q}_{1}=\vec{Q}_{\alpha}-\vec{Q}_{1} \cdot\left(\vec{Q}_{\alpha}\right), \vec{Q}_{2}=\vec{Q}_{\beta}-\vec{B}_{2} \cdot\left(\vec{E}_{\beta}\right), \vec{Q}_{3}=\vec{Q}_{\delta}-\vec{B}_{3} \cdot\left(\vec{E}_{\delta}\right)
\end{aligned}
$$

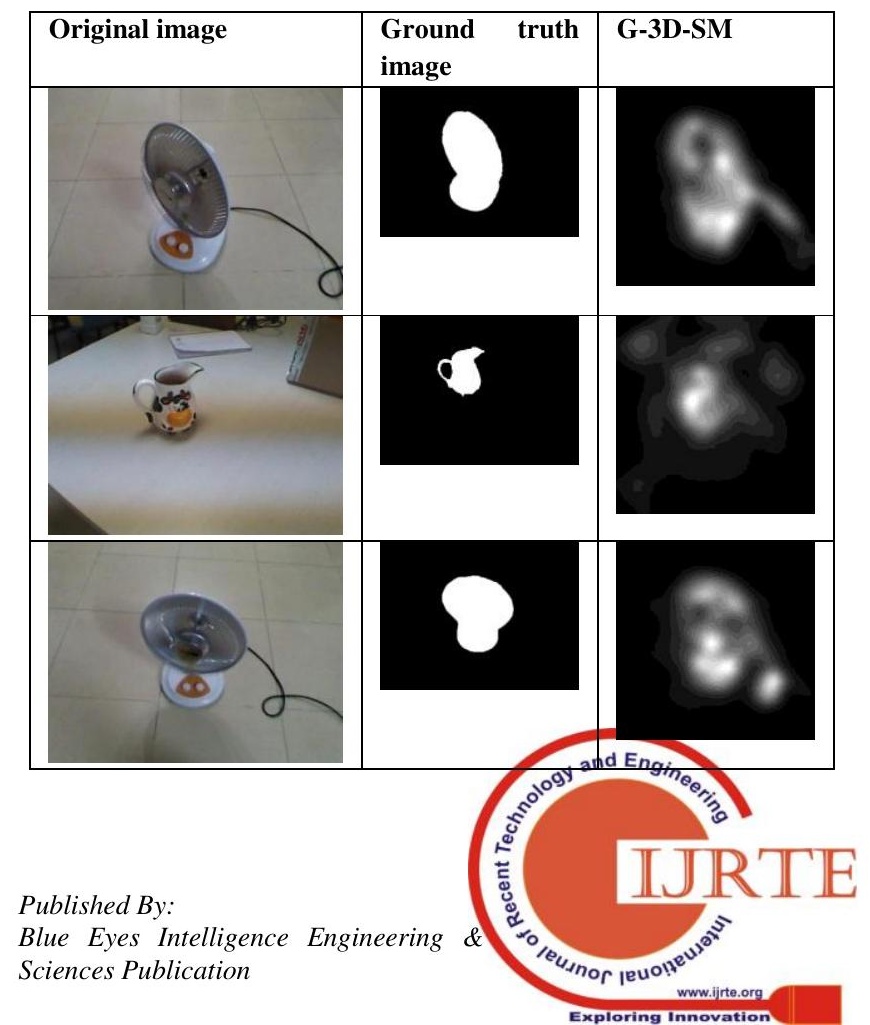




$$
\vec{Q}(i t+1)=\frac{\vec{Q}_{1}+\vec{Q}_{2}+\vec{Q}_{3}}{3}
$$

The last position of $\alpha, \beta$ and $\delta$ indicates the random position in the search space. The pseudo code of GWO is given in Algorithm 1.

In the proposed model, the analysis is held by varying the $a$ 's value from 0.5 to 2.5 , i.e., $0.5,1,1.5,2$ and 2.5 and the corresponding outcomes are attained.

\begin{tabular}{|l|}
\hline \multicolumn{1}{|c|}{ Algorithm 1: GWO Optimization } \\
\hline Initialization of population $Q_{j}(j=1,2, \ldots . . m)$ \\
Initialize $a, B, F$ \\
Fitness evaluation of every search agent \\
$Q_{\alpha}$ indicates best search agent \\
$Q_{\beta}$ denotes the second best search agent \\
$Q_{\delta}$ indicates third best agent \\
While $\left(i t<M a x^{i t}:\right.$ Max ${ }^{i t} \rightarrow$ Maximum iteration) \\
For(each search agent) \\
$\quad$ Position update of search agent by Eq. (16) \\
End for \\
Update $a, B$ and $F$ \\
Fitness evaluation of each search agent \\
Update $Q_{\alpha}, Q_{\beta}$ and $Q_{\delta}$ \\
it $=i t+1$ \\
End while \\
Return $Q_{\alpha}$
\end{tabular}

\subsection{RESUltS AND DisCuSSIONS}

\subsection{Experimental setup}

\subsection{Performance Evaluation}

The performance evaluation of adopted SD model is portrayed by Figure. 5 and Figure. 6 for Gaze and RGDB databases. Accordingly, five sample images from both Gaze and RGDB databases are exploited for examining in respect of ROC. Here, Figure 5 elucidates the assessment of adopted work for Gaze database. Figure. 5(a) delineates that when the TPR is set at
The implemented SD approach was implemented in MATLAB 2015a using two databases namely, 3DGaze database and RGBD database. RGBD database includes 135 images, in which 35 images were training images, and residual 100 images were testing images. In 3DGaze database, there were 18 images, in which 10 images and 8 images were training images and testing images, respectively. Moreover, the outcomes of varying $a$ were also analyzed with respect to the False Positive Rate (FPR) value and True Positive Rate (TPR) value. The input and output image of both Gaze database and RGBD database is evinced in Figure 4 and Figure. 3, respectively.

Figure 3: Input and Output Images of traditional models and Proposed model using RGBD Database

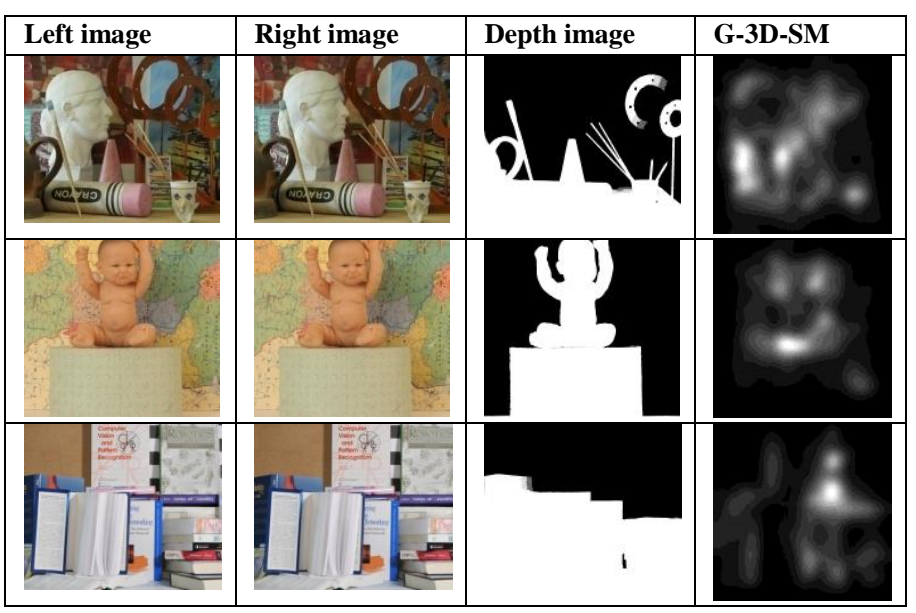

Figure 4: Input and Output Images of traditional models and Proposed model using 3DGAZE Database

1, the FPR of $a=1.5$ is $4.17 \%$ better than $a=0.5, a=1$, $a=2$ and $a=2.5$. Similarly, from Figure. 5(d), when the TPR is set at 1 , the FPR of $a=2.5$ is $2.22 \%$ superior to $a=0.5, a=1, a=1.5$ and $a=2$. Also, from Figure. 6(b), when the TPR is set at 1 , the FPR of $a=2$ is $2.22 \%$ superior to $a=0.5, a=1.5$ and $a=2$ and is $4.54 \%$ superior to $a=1$. Thus, the performance analysis of the adopted model using Gaze and RGDB databases has been proved effective. 
Grey Wolf Optimization on Modeling Saliency Detection in Stereoscopic 3D Images

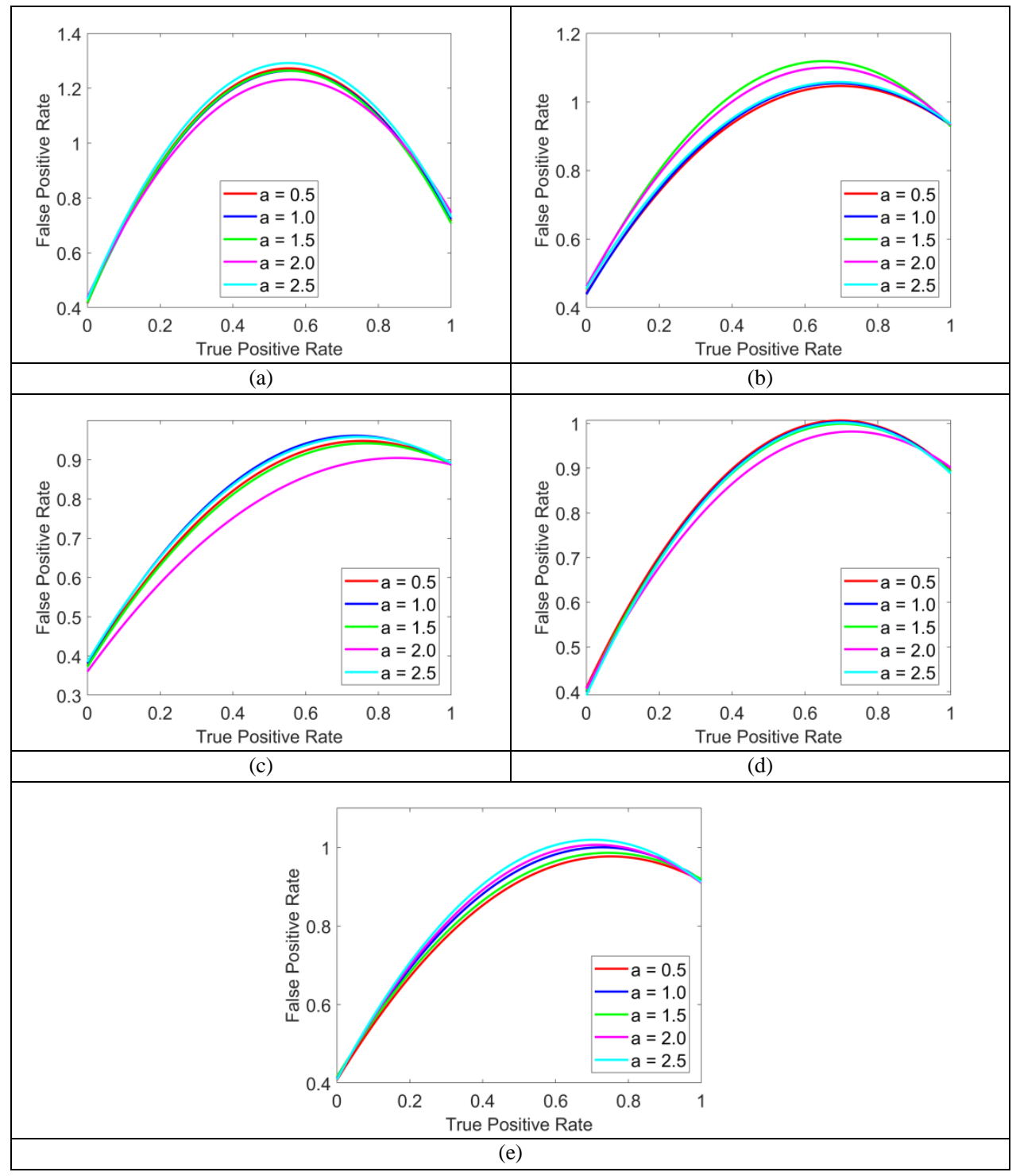

Figure 5: Performance analysis in terms of ROC of proposed model by considering 5 samples using Gaze database (a) sample 1(b) sample 2 (c) sample 3 (d) sample 4 (e) sample 5

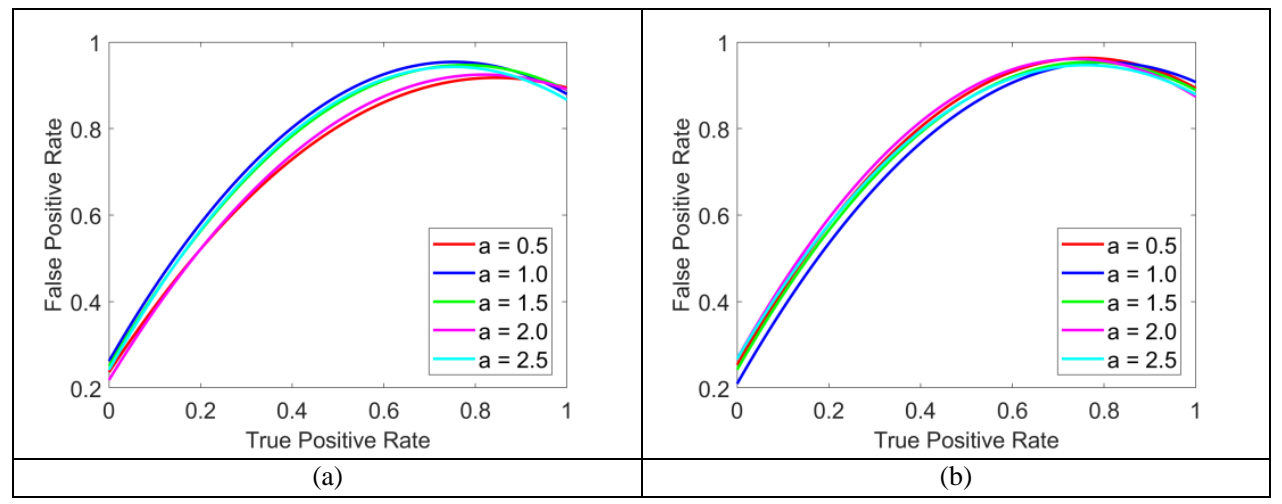




\section{Grey Wolf Optimization on Modeling Saliency Detection in Stereoscopic 3D Images}

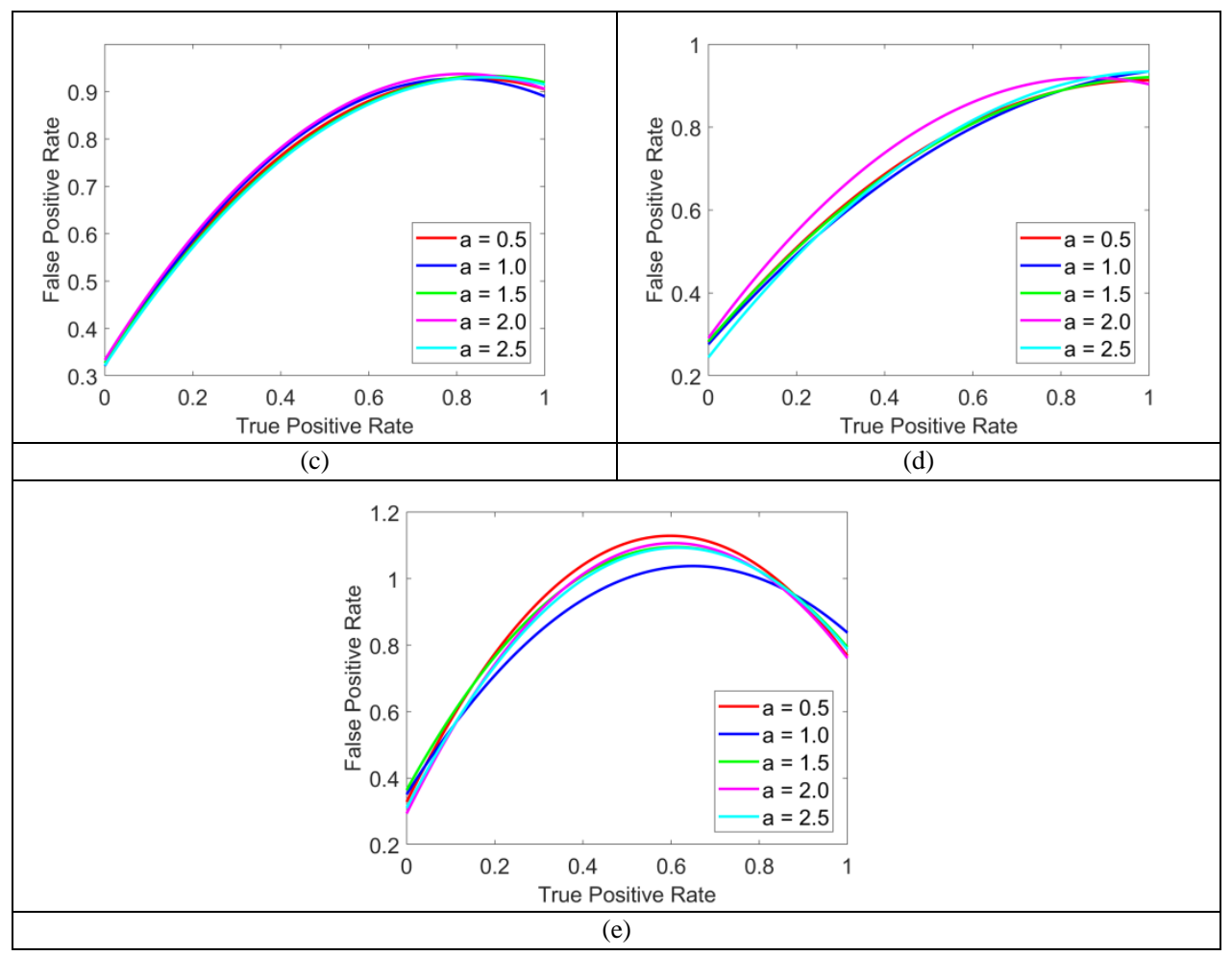

Figure 6: Performance analysis in terms of ROC of proposed model by considering 5 samples using RGDB database (a) sample 1(b) sample 2 (c) sample 3 (d) sample 4 (e) sample 5

\subsection{Statistical Analysis}

As the meta-heuristic algorithms are stochastic in nature, accurate results could not be attained. Hence, all the algorithms are done five times and best, mean, worst, standard deviation and median is determined. From Table. 1, the best performance at $a=1.5$ is $9.01 \%$ better than $a=0.5$, $3.15 \%$ better than $a=1,1.85 \%$ better than $a=2$ and $0.3 \%$ better than $a=2.5$. Consequently, the mean performance at $a=0.5$ is $1.06 \%$ superior to $a=1,3.63 \%$ better than $a=1.5$, $5.72 \%$ superior to $a=2$ and $5.53 \%$ superior to $a=2.5$. From Table. 2, the median performance at $a=1.5$ is $0.75 \%$ better than $a=0.5,2.26 \%$ better than $a=1,10.72 \%$ better than $a=2$ and $10.36 \%$ better than $a=2.5$. Also, from Table. 4 , the best performance at $a=2$ is $5.6 \%$ better than $a=0.5,2.6 \%$ better than $a=1,0.15 \%$ better than $a=1.5$ and $0.14 \%$ superior to $a=2.5$. In the same way, from Table. 5, the worst performance at $a=2$ is $10.10 \%$ superior to $a=0.5,8.8 \%$ superior to $a=1,2.43 \%$ superior to $a=1.5$ and $4.16 \%$ superior to $a=2.5$. Also, Table. 6 precisely delineates that the performance of standard deviation at $a=2$ is $2.14 \%$ better than $a=0.5,3.24 \%$ better than $a=1,1.85 \%$ better than $a=1.5$ and $12.76 \%$ better than $a=2.5$.

Table 1: Statistical Analysis Of Proposed Model Regarding Pcc Using Rgbd Database

\begin{tabular}{|l|r|r|r|r|r|}
\hline Statistics & \multicolumn{1}{|l|}{$a=0.5$} & \multicolumn{1}{l|}{$a=1$} & \multicolumn{1}{l|}{$a=1.5$} & \multicolumn{1}{l|}{$a=2$} & \multicolumn{1}{l|}{$a=2.5$} \\
\hline Best & 0.88699 & 0.88751 & 0.88779 & 0.87169 & 0.88509 \\
\hline Worst & -0.00573 & 0.021585 & 0.004549 & 0.030113 & -0.0237 \\
\hline Mean & 0.52344 & 0.51789 & 0.50443 & 0.4935 & 0.49449 \\
\hline Median & 0.54269 & 0.54223 & 0.52464 & 0.50056 & 0.50453 \\
\hline Standard deviation & 0.18133 & 0.17748 & 0.17243 & 0.1707 & 0.17513 \\
\hline
\end{tabular}


Grey Wolf Optimization on Modeling Saliency Detection in Stereoscopic 3D Images

Table 2: Statistical analysis of proposed model regarding KLD using RGBD database

\begin{tabular}{|l|r|r|r|r|r|}
\hline Statistics & \multicolumn{1}{|l|}{$a=0.5$} & \multicolumn{1}{|l|}{$a=1.5$} & \multicolumn{1}{l|}{$a=2$} & \multicolumn{1}{l|}{$a=2.5$} \\
\hline Best & 0.1379 & 0.10378 & 0.087081 & 0.11751 & 0.14123 \\
\hline Worst & 1.7933 & 1.8274 & 1.8665 & 1.7867 & 1.7989 \\
\hline Mean & 0.63812 & 0.63536 & 0.63833 & 0.65396 & 0.64401 \\
\hline Median & 0.49241 & 0.50006 & 0.48873 & 0.54745 & 0.54523 \\
\hline Standard deviation & 0.38056 & 0.38495 & 0.39039 & 0.38284 & 0.38311 \\
\hline
\end{tabular}

Table 3: Statistical analysis of proposed model regarding AUC(RGBD database)

\begin{tabular}{|l|r|r|r|r|r|}
\hline Statistics & \multicolumn{1}{|l|}{$a=0.5$} & \multicolumn{1}{|l|}{$a=1$} & $a=1.5$ & \multicolumn{1}{l|}{$a=2$} & \multicolumn{1}{l|}{$a=2.5$} \\
\hline Best & 0.98929 & 0.99513 & 0.99059 & 0.99896 & 0.99887 \\
\hline Worst & 0.67427 & 0.68686 & 0.68918 & 0.68632 & 0.67764 \\
\hline Mean & 0.87959 & 0.87966 & 0.87931 & 0.88201 & 0.88018 \\
\hline Median & 0.89149 & 0.88948 & 0.89113 & 0.88547 & 0.884 \\
\hline Standard deviation & 0.065113 & 0.064525 & 0.063412 & 0.062373 & 0.064552 \\
\hline
\end{tabular}

Table 4: Statistical analysis of proposed model regarding PCC using Gaze database

\begin{tabular}{|l|r|r|r|r|r|}
\hline Statistics & \multicolumn{1}{|l|}{$a=0.5$} & \multicolumn{1}{|l|}{$a=1.5$} & $a=2$ & \multicolumn{1}{l|}{$a=2.5$} \\
\hline Best & 0.9981 & 0.9984 & 0.99717 & 0.99866 & 0.99722 \\
\hline Worst & 0.84508 & 0.85285 & 0.85974 & 0.87104 & 0.86702 \\
\hline Mean & 0.96334 & 0.96386 & 0.96397 & 0.96367 & 0.9631 \\
\hline Median & 0.98136 & 0.98127 & 0.97928 & 0.98263 & 0.98027 \\
\hline Standard deviation & 0.041592 & 0.040252 & 0.038177 & 0.038297 & 0.0382 \\
\hline
\end{tabular}

Table 5: Statistical analysis of proposed model regarding kld using Gaze database

\begin{tabular}{|l|r|r|r|r|r|}
\hline Statistics & \multicolumn{1}{|l|}{$a=0.5$} & \multicolumn{1}{|l|}{$a=1$} & $a=1.5$ & \multicolumn{1}{l|}{$a=2$} & $a=2.5$ \\
\hline Best & 0.00171 & 0.001746 & 0.00175 & 0.001341 & 0.001571 \\
\hline Worst & 0.10151 & 0.10007 & 0.093529 & 0.091256 & 0.095217 \\
\hline Mean & 0.021385 & 0.019697 & 0.018502 & 0.018814 & 0.018546 \\
\hline Median & 0.011451 & 0.009604 & 0.009198 & 0.009151 & 0.008972 \\
\hline Standard deviation & 0.026109 & 0.025873 & 0.02354 & 0.023148 & 0.023931 \\
\hline
\end{tabular}

Table 6: Statistical analysis of proposed model regarding AUC using Gaze database

\begin{tabular}{|l|r|r|r|r|r|}
\hline Statistics & \multicolumn{1}{|c|}{$a=0.5$} & \multicolumn{1}{|l|}{$a=1$} & $a=1.5$ & \multicolumn{1}{l|}{$a=2$} & \multicolumn{1}{l|}{$a=2.5$} \\
\hline Best & 0.97293 & 0.98196 & 0.9777 & 0.98225 & 0.97415 \\
\hline Worst & 0.7717 & 0.77169 & 0.77219 & 0.78434 & 0.78262 \\
\hline Mean & 0.90335 & 0.90306 & 0.9046 & 0.90352 & 0.90595 \\
\hline Median & 0.91367 & 0.90826 & 0.9199 & 0.91561 & 0.91489 \\
\hline Standard deviation & 0.061484 & 0.060828 & 0.061659 & 0.062797 & 0.055692 \\
\hline
\end{tabular}




\subsection{CONClusion}

With the intention of overcoming the challenges met with $\mathrm{SD}$, this paper has presented an analysis on the novel SD model using GWO algorithm. The adopted scheme involves two phases: i) FE, which exploits Gaussian kernel design for extracting the features and ii) depth SD, which exploits GF for attaining the depth saliency map. Accordingly, two coefficients such as feature difference betwixt image patches, $H$ from feature assessment, and fine-scale $c$ was optimized to accomplish the accurate detection, and this was attained by a renowned GWO optimization algorithm. Further, the proposed model was evaluated under varying values of $a$.Hence, the overall analysis has proven the enhancement of proposed SD model in terms of ROC and statistical analysis.

\section{REFERENCES}

[1]. Runmin Cong, Jianjun Lei, Changqing Zhang, Qingming Huang, Xiaochun Cao, Chunping Hou, "Saliency Detection for Stereoscopic Images Based on Depth Confidence Analysis and Multiple Cues Fusion", IEEE Journals \& Magazines, Vol.23, no. 6, pp. $819-823,2016$.

[2]. Shiqi Li, Cheng Zeng, Shiping Liu, Yan Fu, "Merging fixation for saliency detection in a multilayer graph", Neurocomputing, Vol. 230, pp. 173-183, 2017.

[3]. Changqing Zhang, Zhiqiang Tao, Xingxing Wei, Xiaochun Cao, "A flexible framework of adaptive method selection for image saliency detection", Pattern Recognition Letters, Vol. 63, pp. 6670, 2015.

[4]. Yanbang Zhang, Fen Zhang, Lei Guo, "Saliency detection by selective colour features", Neurocomputing, Vol. 203, pp. 34-40, 2016.

[5]. W. Diao, X. Sun, X. Zheng, F. Dou, H. Wang and K. Fu, "Efficient Saliency-Based Object Detection in Remote Sensing Images Using Deep Belief Networks", IEEE Geoscience and Remote Sensing Letters, vol. 13, no. 2, pp. 137-141, 2016.

[6]. Yuming Fang, Jianjun Lei, Jia Li, Long Xu, Patrick Le Callet, "Learning visual saliency from human fixations for stereoscopic images", Neuro computing, 2017.

[7]. . Zhengbing Wang, Guili Xu, Zhengsheng Wang, Chunxing Zhu, "Saliency detection integrating both background and foreground information", Neurocomputing, vol. 216, pp. 468-477, 2016.

[8]. Wei Chen, Tongfeng Sun, Ming Li, Haifeng Jiang, Chongbo Zhou, "A new image co-segmentation method using saliency detection for surveillance image of coal miners", Computers \& Electrical Engineering, vol. 40, no. 8, pp. 227-235, 2014.

[9]. Jinqing Qi, Shijing Dong, Fang Huang, Huchuan Lu, "Saliency detection via joint modeling global shape and local consistency", Neurocomputing, vol. 222, pp. 81-90, 2017.

[10]. Yuming Fang, Junle Wang, Yuan Yuan, Jianjun Lei, Patrick Le Callet, "Saliency-based stereoscopic image retargeting", Information Sciences, vol. 372, pp. 347-358, 2016.
[11]. Yun Liu, Jiachen Yang, Qinggang Meng, Zhihan Lv, Zhiqun Gao, "Stereoscopic image quality assessment method based on binocular combination saliency model", Signal Processing, vol. 125, pp. 237-248, 2016.

[12]. Qiuping Jiang, Feng Shao, Gangyi Jiang, Mei Yu, Changhong $\mathrm{Yu}$, "A depth perception and visual comfort guided computational model for stereoscopic 3D visual saliency", Signal Processing: Image Communication, vol. 38, pp. 57-69, 2015.

[13]. Xudong Zhang, Yi Wang, Jun Zhang, Liangmei Hu, Meng Wang, "Light field saliency vs. 2D saliency: A comparative study", Neuro computing, vol. 166, pp. 389-396, 2015.

[14]. Gangyi Jiang, Haiyong Xu, Mei Yu, Ting Luo, Yun Zhang, "Stereoscopic image quality assessment by learning non-negative matrix factorization-based color visual characteristics and considering binocular interactions" Journal of Visual Communication and Image Representation, vol.46, pp. 269-279, 2017.

[15]. Yuming Fang; Junle Wang; Manish Narwaria; Patrick Le Callet; Weisi Lin, "Saliency Detection for Stereoscopic Images", IEEE Journals \& Magazines, vol. 23, no. 6, pp. 2625 - 2636, 2014.

[16]. Hangke Song; Zhi Liu; Huan Du; Guangling Sun; Olivier Le Meur; Tongwei Ren, "Depth-Aware Salient Object Detection and Segmentation via Multiscale Discriminative Saliency Fusion and Bootstrap Learning", IEEE Journals \& Magazines, vol. 26, no. 9, pp. 4204 - 4216, 2017.

[17]. Huan Du; Zhi Liu; Hangke Song; Lin Mei; Zheng Xu, "Improving RGBD Saliency Detection Using Progressive Region Classification and Saliency Fusion”, IEEE Journals \& Magazines, vol. 4, pp. 8987 - 8994, 2016.

[18]. Wenguan Wang; Jianbing Shen; Yizhou Yu; Kwan-Liu Ma, "Stereoscopic Thumbnail Creation via Efficient Stereo Saliency Detection", IEEE Journals \& Magazines, vol. 23, no. 8, pp. 2014 - 2027, 2017.

[19]. Renbin Peng, Pramod K. Varshney, “ A human visual systemdriven image segmentation algorithm", Journal of Visual Communication and Image Representation, vol. 26, pp.66-79, 2015. 


\section{Author Biography}

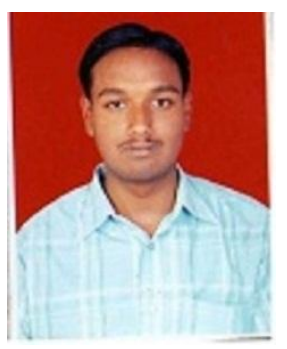

Mr.Rakesh Y, received his B.Tech. degree from department of ECE at JNTU, Hyderabad in 2006. M.Tech degree from JNTU, Kakinada in 2010. He is currently working as assistant professor in the department of ECE at Usha Rama College of Engineering and Technology (URCET) Telaprolu, near Vijayawada city in Andhra Pradesh. He is pursuing his $\mathrm{PhD}$ degree from Acharya Nagarjuna University, Guntur, Andhra Pradesh, India. His current research interests include Image and signal processing, Pattern recognition, Computer vision

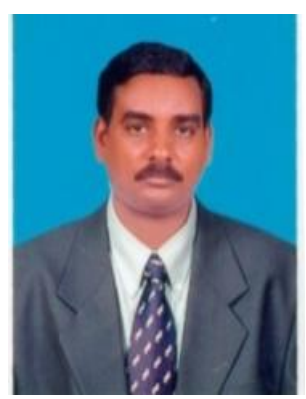

Dr.K Sri Rama Krishna received his B.E degree from ECE department of JNTU, Kakinada in 1986.M.E degree from Birla Institute of Technology and Science Pilani, in 1992. Ph.D. from ECE department of Andhra University, Visakhapatnam, Waltair Junction, Visakhapatnam, Andhra Pradesh. He is Currently working as Professor and Head of ECE Department at Velagapudi Ramakrishna Siddhartha Engineering College (VRSEC), Chalasani Nagar, Kanuru, Vijayawada, Andhra Pradesh in 2002. His current research interests include ANN, Image and signal processing, Microwave, Antennas. 